\title{
Cosmic Rays: A View Into Galactic Interactions and the New Physics
}

\section{Tijana Prodanović ${ }^{* \dagger}$}

Department of Physics, University of Novi Sad, Serbia

E-mail: prodanvc@df.uns.ac.rs

\section{Darko Donevski}

Department of Physics, University of Novi Sad, Serbia

E-mail: darko.donevski@df.uns.ac.rs

\begin{abstract}
It is known that close galactic fly-bys and interactions give rise to shock waves that disrupt the interstellar medium of galaxies and impact their morphologies. These large-scale shocks that form in the interstellar medium of interacting systems will be the sites of particle acceleration giving rise to a population of tidal cosmic rays, in addition to standard galactic cosmic rays present in star-forming galaxies. Abundance measurements in such systems can help us probe the presence of this cosmic-ray population and in turn test the long-lasting primordial lithium problem and the need for the new physics. Such additional cosmic-ray population will also result in non-thermal radiation which will affect the radio brightness of these galaxies and in turn impact the wellestablished far infrared-radio correlation, standardly used as a star-formation rate tracer, among other things. Finally, we will discuss how radio and infrared signatures of these cosmic rays can in turn be used as indicators of galactic interactions which could be especially powerful for minor mergers and at high redshifts.
\end{abstract}

The 34th International Cosmic Ray Conference,

30 July- 6 August, 2015

The Hague, The Netherlands

\footnotetext{
${ }^{*}$ Speaker.

The work of TP is supported in part by the Ministry of Science of the Republic of Serbia under project numbers 171002 and 176005.
} 


\section{Introduction}

It is known that galactic interactions result in large-scale shock waves that impact the interstellar medium [1,2] and can affect the evolution of galaxies [3] and trigger star-formation [4]. However, it was pointed out [5] that galactic interactions can also be a new source of cosmic rays accelerated [6] in large-scale tidal shocks âĂŞ tidal cosmic rays (TCRs). The possible effects of TCRs have been explored by looking at how they would impact light element abundances [5] and non-thermal radio emission [7] of interacting galaxy. Both studies have demonstrated that these effects can be significant.

Besides electromagnetic radiation, any cosmic-ray population will also be involved in the nucleosynthesis of light elements [8]. Especially interesting is the case of lithium that was recently measured in the interstellar medium (ISM) of the Small Magellanic Cloud (SMC) [9]. Prodanovic et al. 2013 have pointed out that it would be sufficient for the entire gas of the SMC to be shocked only twice, to accelerate enough TCRs to produce as much lithium as galactic cosmic-rays (GCRs) have made throughout its history. This is consistent with the fact that SMC has already experienced at least two close galactic fly-bys and with the anomalously high observed abundance ratio between the two lithium isotopes [5]. Therefore, observations of lithium abundance in the SMC indicate that additional cosmic-ray population may have been produced at some epoch.

When galaxies with active star-formation are observed it is generally considered that observed far-infrared (FIR) emission originates dominantly from dust heated by the UV radiation from young massive stars, while the diffuse radio emission of the galaxy comes from non-thermal synchrotron emission of GCR electrons accelerated in supernova remnants. Since all of these have essentially the same source in massive stars, it was expected and found that there exists a tight relation between FIR and radio luminosities of star-forming galaxies [10,11]. This FIR-radio correlation has been found to hold for redshifts from 0 to 2 for various types of galaxies $[12,13]$ and has ever since been a powerful tool [14] for determining star-formation rates of galaxies [15], and star-formation history of galaxies. However, if a significant flux of tidal-cosmic rays is present, compared to galactic cosmic-ray flux, its presence would impact the FIR-radio correlation and consequently impact our understanding of the star-formation history of the interacting galaxy.

\section{Radio and Infrared Emission of Interacting Galaxies}

To explore the possibility of tidal cosmic-ray presence in interacting galaxies, in Donevski Prodanovic 2015 we have analysed a sample of 43 available $[16,17,18]$ interacting galaxies in various merger stages, and explored possible differences in their radio and FIR emission spectra and luminosities. The sample, along with its properties and references is presented in Table 1. Specifically, we explored the effects that the tidal shock heating and particle acceleration might have had on the FIR-radio correlation and radio spectra of the star-forming galaxies. The FIRradio correlation is described using the $q_{\mathrm{IR}}$ parameter [19] as

$$
q_{\mathrm{IR}}=\log \left(\frac{F_{\mathrm{FIR}}}{3.75 \times 10^{12} \mathrm{Wm}^{-2}}\right)-\log \left(\frac{S_{1.4}}{\mathrm{Wm}^{-2} \mathrm{~Hz}^{-1}}\right)
$$

where $S_{1.4}$ is the continuum radio emission flux at $1.4 \mathrm{GHz}$ per frequency such that $S_{1.4} \propto v^{-\alpha}$ and $\alpha$ is the radio spectral index, positive in majority of sources, while $F_{\mathrm{FIR}}$ is the rest-frame FIR 


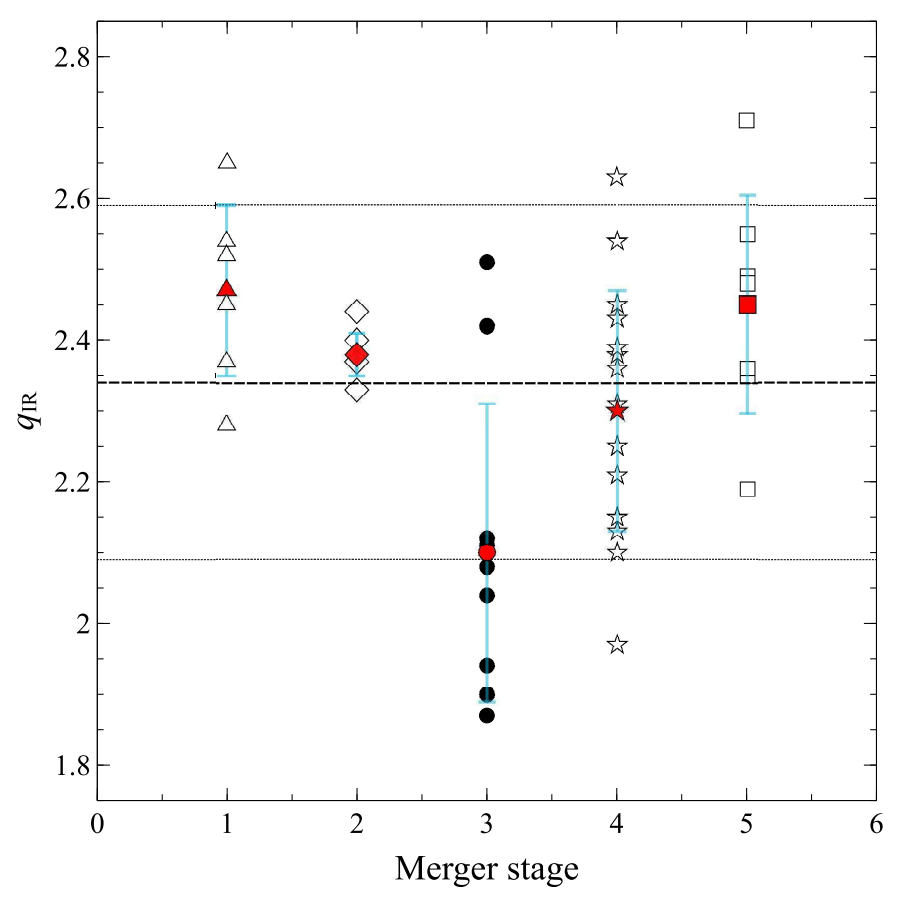

Figure 1: FIR-radio parameter $q_{\mathrm{IR}}$ for 43 interacting star-forming galaxy pairs at different merger stages according to [20] classification. The dashed line is the typical value of the $q_{\mathrm{IR}}=2.34 \pm 0.21$ [11], with mean deviation denoted with dotted lines. Mean FIR-radio parameter was also determined for each merger stage separately and presented as filled (red) symbols with blue error bars. This plot was presenter in [7].

dust emission flux. Analysing this sample of interacting galaxies we found that it behaved as it would be qualitatively expected [7] if there was significant impact of tidal shocks on the ISM: early merger stages would be dominated by additional heating due to tidal shocks (increasing FIRradio parameter $q_{I R}$ ), followed by the increase in non-thermal radiation as TCRs get accelerated (decreasing $q_{I R}$ ), and eventually star-formation would be enhanced and so the effects of massive stars would again become dominant (increasing $q_{I R}$ ). This trend is seen on Figure 1 [7] where FIRradio parameter as a function of different merger stages ( 0 being no merger, 6 being post-merger system) according to [20]. We see that the FIR-radio correlation parameter, varies across different merger stages of interacting galaxies differently, but in a manner that would be expected under the hypothesis of tidal shock heating and particle acceleration [7]. Thus, FIR and radio observations indicate that a new cosmic-ray population is potentially present in interacting galaxies.

Further-more we have also explored how the spectral index varies across different merger stages and results are presented on Figure 2 [7]. What would be expected if an additional cosmicray population is present is enhanced non-thermal emission resulting in a spectral index being steeper than the typical value (larger value of $\alpha$ ). We would expect this to be most pronounced in the middle phase of interaction (merger stage 3) where the FIR-radio parameter also deviates most from its typical value. What we see on Fig. 2 is that most of the merger 3 stage systems (filled circles) cluster in the upper left quadrant of the plot corresponding to spectral index values larger than the typical value. However, to confirm this with more accuracy one would need a larger sample due to present dispersion. 


\begin{tabular}{|c|c|c|c|c|c|c|c|}
\hline IRAS name & Merger stage & $\operatorname{SFR}(1.4 \mathrm{GHz})$ & $\operatorname{SFR}(\mathrm{H} \alpha)$ & SFR(IR) & $q_{\mathrm{IR}}$ & $\alpha$ & $\Delta\left(q_{\mathrm{IR}}\right)$ \\
\hline $00085-1223$ & $\overline{5}$ & 31.53 & 1.05 & 51.6 & 2.48 & 0.70 & -0.14 \\
\hline 01053-1746 & 3 & 119.1 & 17.19 & 44.8 & 2.08 & 0.90 & 0.26 \\
\hline $01173+1405$ & 2 & 59.35 & 0.75 & 83.6 & 2.40 & 0.69 & -0.06 \\
\hline 01341-3734 & 3 & 11.6 & 1.46 & 7.11 & 1.90 & 0.82 & 0.44 \\
\hline 01364-1042 & 5 & 46.17 & 1.11 & 118.0 & 2.71 & 0.24 & -0.37 \\
\hline $02512+1446$ & 2 & 59.50 & 5.42 & 76.11 & 2.33 & 0.33 & 0.01 \\
\hline 04131-2836 & 4 & 13.42 & 26.7 & 8.67 & 2.10 & 0.90 & 0.24 \\
\hline 04315-0840 & 5 & 36.73 & 58.0 & 67.3 & 2.49 & 0.53 & -0.15 \\
\hline 04591-0419 & 4 & 6.02 & 5.86 & 5.93 & 2.25 & 0.70 & 0.09 \\
\hline 04595-1813 & 3 & 18.78 & 8.86 & 6.06 & 1.87 & 1.42 & 0.47 \\
\hline 06295-1735 & 3 & 11.41 & 2.28 & 20.8 & 2.42 & 1.09 & -0.08 \\
\hline $08087+0347$ & 1 & 3.52 & 0.33 & 5.73 & 2.45 & 0.51 & -0.11 \\
\hline 08175-1433 & 4 & 21.07 & 4.56 & 15.1 & 2.13 & 0.96 & 0.21 \\
\hline 09006-6404 & 4 & 12.2 & 5.40 & 15.4 & 2.38 & 0.61 & -0.04 \\
\hline $09126+4432$ & 2 & 77.69 & 3.11 & 95.7 & 2.37 & 0.34 & -0.03 \\
\hline $09437+0317$ & 3 & 20.1 & 13.43 & 20.1 & 2.04 & 0.92 & 0.30 \\
\hline $10015-0614$ & 3 & 37.5 & 17.5 & 27.4 & 2.12 & 0.76 & 0.22 \\
\hline $10565+2448$ & 2 & 147.65 & 109.1 & 208.2 & 2.44 & 0.73 & -0.10 \\
\hline 11005-1601 & 1 & 12.35 & 15.3 & 11.2 & 2.28 & 0.98 & 0.06 \\
\hline $11010+4107$ & 2 & 40.06 & 2.15 & 79.11 & 2.37 & 0.72 & -0.03 \\
\hline $11122-2327$ & 5 & 10.8 & 3.58 & 14.4 & 2.35 & 0.60 & -0.1 \\
\hline $11231+1456$ & 1 & 32.24 & 2.17 & 72.5 & 2.65 & 0.47 & -0.31 \\
\hline $11396+0036$ & 5 & 11.86 & 6.59 & 10.9 & 2.19 & 0.30 & 0.11 \\
\hline 11409-1631 & 5 & 4.89 & 1.60 & 4.45 & 2.55 & 0.58 & -0.21 \\
\hline $12120-1118$ & 1 & 13.9 & 6.45 & 10.9 & 2.37 & 0.77 & -0.03 \\
\hline $12286-2600$ & 3 & 7.07 & 22.7 & 13.4 & 2.51 & 0.89 & -0.17 \\
\hline $12596-1529$ & 4 & 9.28 & 7.80 & 15.4 & 2.54 & 0.66 & -0.20 \\
\hline $13183+3423$ & 4 & 82.25 & 11.4 & 79.25 & 2.33 & 0.50 & 0.01 \\
\hline $13362+4831$ & 3 & 8.94 & 62.09 & 123.02 & 1.90 & 0.72 & 0.44 \\
\hline $13373+0105$ & 4 & 22.6 & 44.3 & 46.6 & 2.45 & 0.62 & -0.11 \\
\hline $14150-0711$ & 4 & 2.27 & 2.18 & 3.01 & 2.39 & 0.72 & -0.05 \\
\hline $14423-2042$ & 3 & 12.8 & 5.86 & 11.41 & 2.11 & 0.82 & 0.23 \\
\hline $15163+4255$ & 4 & 145.98 & 4.72 & 141.45 & 2.36 & 0.72 & -0.02 \\
\hline $15172-3115$ & 5 & 2.73 & 6.01 & 6.67 & 2.36 & 0.63 & -0.02 \\
\hline $16577+5900$ & 3 & 1.19 & 39.81 & 345.07 & 1.94 & 0.88 & 0.40 \\
\hline $18093-5744$ & 4 & 32.01 & 21.2 & 39.06 & 2.43 & 0.93 & -0.09 \\
\hline $18341-5732$ & 1 & 15.51 & 55.9 & 28.1 & 2.54 & 0.76 & -0.21 \\
\hline
\end{tabular}




\begin{tabular}{cccccccc} 
IRAS name & Merger stage & SFR $(1.4 \mathrm{GHz})$ & SFR $(\mathrm{H} \alpha)$ & SFR(IR) & $q_{\mathrm{IR}}$ & $\alpha$ & $\Delta\left(q_{\mathrm{IR}}\right)$ \\
\hline \hline $20305-0211$ & 4 & 45.12 & 9.29 & 21.8 & 1.97 & 0.79 & 0.37 \\
$21330-3846$ & 4 & 7.95 & 5.47 & 18.1 & 2.63 & 0.56 & -0.29 \\
$23157-0441$ & 4 & 47.28 & 76.1 & 36.1 & 2.15 & 1.05 & 0.19 \\
$23336+0152$ & 4 & 6.04 & 4.45 & 7.91 & 2.31 & 0.67 & 0.03 \\
$23394-0353$ & 4 & 27.65 & 36.5 & 22.8 & 2.21 & 0.82 & 0.13 \\
$23488+2018$ & 1 & 29.58 & 2.29 & 53.27 & 2.52 & 0.67 & -0.14 \\
\hline
\end{tabular}

Table 1: Properties of the Sample Galaxies. References: [16], [17], [18]. Notes: SFR $(1.4 \mathrm{GHz})=5.52 \times$ $10^{-22} \times L_{1.4 \mathrm{GHz}}\left[M_{\odot} \mathrm{yr}^{-1}\right] ; L_{1.4 \mathrm{GHz}}=4 \pi D^{2} \times S_{1.4 \mathrm{GHz}}\left[\mathrm{WHz}^{-1}\right] . \quad \operatorname{SFR}(\mathrm{H} \alpha)=5.5 \times 10^{-42} \times L_{\mathrm{H} \alpha}\left[\mathrm{M}_{\odot} \mathrm{yr}^{-1} ;\right.$ $L_{\mathrm{H} \alpha}=4 \pi D^{2} \times I_{\mathrm{H} \alpha} \times 10^{0.4 A_{v}}\left[\mathrm{WHz}^{-1}\right] ; \Delta\left(q_{\mathrm{IR}}\right)=q_{0, \mathrm{IR}}-q_{\mathrm{IR}}$, where $q_{0, \mathrm{IR}}$ is the nominal value from [11], while $q_{\mathrm{IR}}$ is the value for a given source.

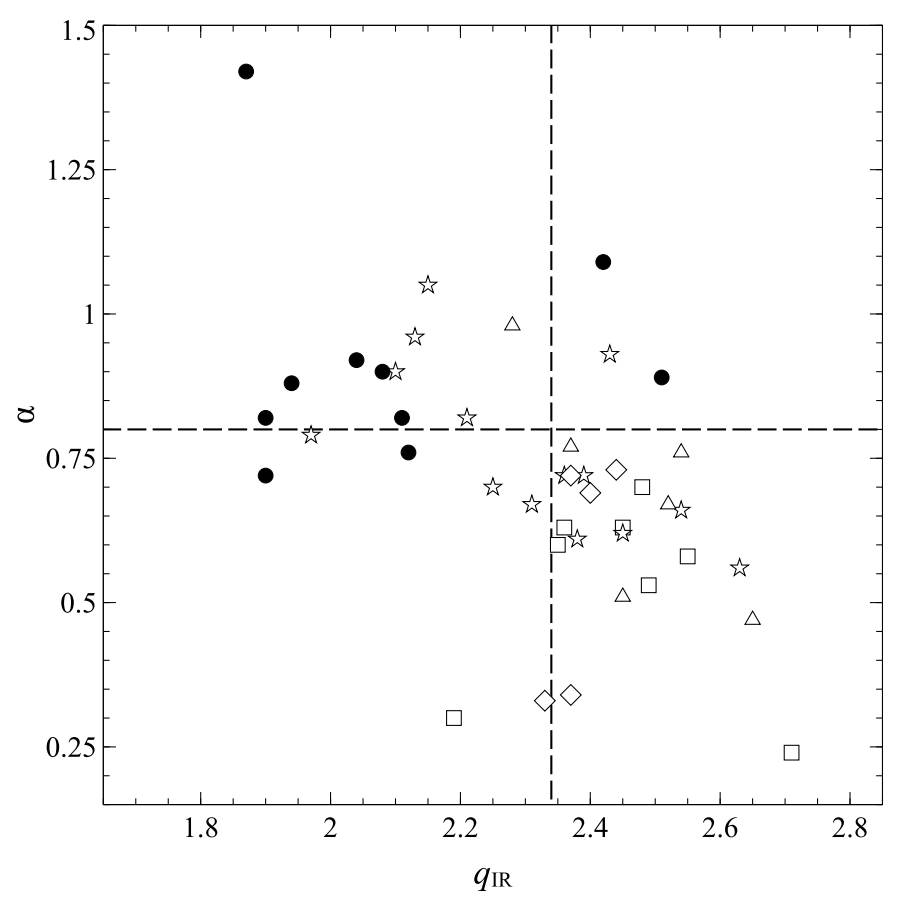

Figure 2: Radio spectral index $\alpha\left(S_{1.4} \propto v^{-\alpha}\right)$ measured between $1.4 \mathrm{GHz}$ and $4.8 \mathrm{GHz}$, as a function of the $q_{\mathrm{IR}}$ parameter for the same sample of interacting star-forming galaxies. Different merger stages are represented with same symbols as in Fig. 1. Typical values of radio spectral index $\alpha=0.8$ [14] and $q_{\mathrm{IR}}$ [11] are represented with dashed lines. This plot was presented in [7].

On important consequence of galactic interaction could also be the impact it has on starformation rate of the galaxy. It is already known that galactic fly-bys and merger events can and do trigger star-formation so that events of galactic interactions are followed by episodes of enhanced star-formation. Star-formation rate is determined indirectly from tracers. One of the well-established tracers of the star-formation rate (SFR) is the $\mathrm{H} \alpha$ emission of a galaxy [21], since the $\mathrm{H} \alpha$ emission traces $\mathrm{HII}$ regions around young $\mathrm{O}$ and $\mathrm{B}$ type stars. Other tracers used to determine SFR are the infrared emission of the heated dust and radio observations at $1.4 \mathrm{GHz}$ $[22,23,24]$. Radio continuum emission from star forming and starburst galaxies originates from 


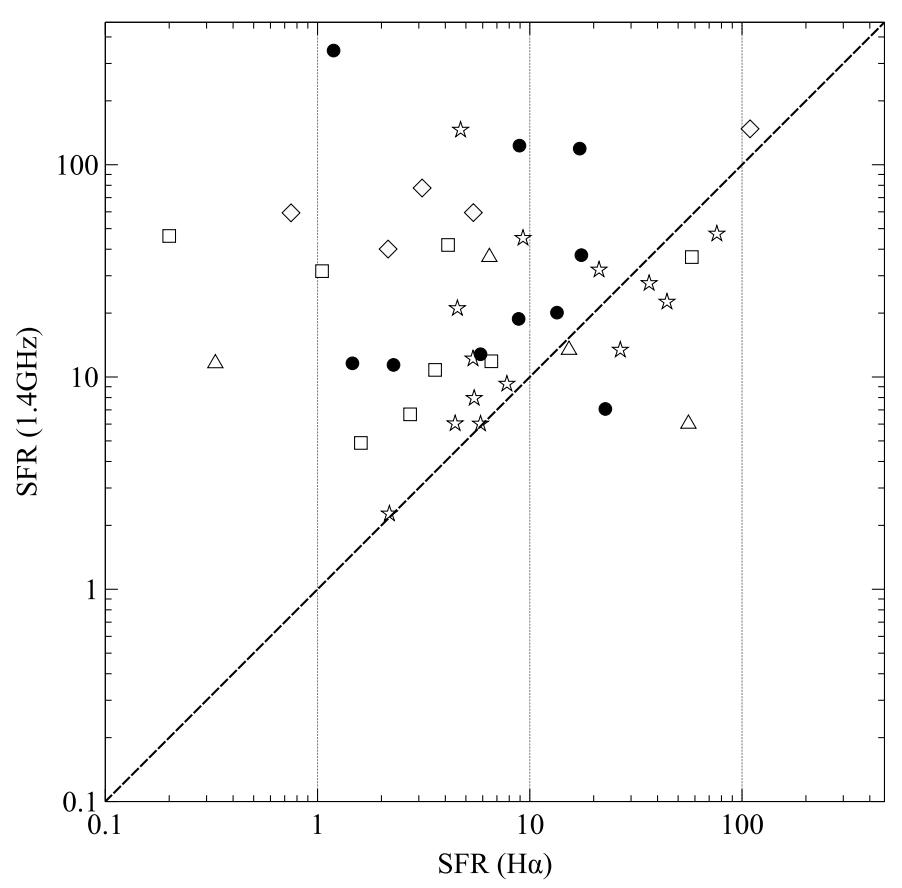

Figure 3: Star-formation rate determined from $\mathrm{H} \alpha$ flux compared to star-formation rate determined from IR flux. This is a preliminary result.

two main sources. The dominant component at the frequencies that are usually observed in this context (e.g. $1.4 \mathrm{GHz}$ ) is the synchrotron emission from cosmic-ray electrons. A shallower thermal component is also present due to bremsstrahlung (free-free) radiation from electrons in HII regions, but this becomes dominant at high frequencies (higher than $30 \mathrm{GHz}$ ) and at $1.4 \mathrm{GHz}$ only comprises $\sim 10 \%$ of the radio flux [14]. However, radio star-formation estimators are calibrated to the IR, via the FIR-radio correlation [15]. From this it is clear that the presence of tidal shocks could greatly impact SFR determined from the FIR and radio emission as tracers if they cause a significant dispersion in the FIR-radio correlation. If tidal shocks have provided extra heating of the dust (expected of early phases), the SFR determined from the IR emission of the dust will be overestimated and greater than SFR determined from $\mathrm{H} \alpha$ emission, while if there was a significant flux of tidal cosmic-rays (expected of mid phase) the SFR determined from $1.4 \mathrm{GHz}$ observations would be overestimated. To test this we compare SFR determined from $\mathrm{H} \alpha$ emission with the one determined from radio observations. We plot these preliminary results in Figure 3. We see that in general, SFR determined from radio observations that are calibrated by the FIR-radio correlation result in higher star-formation rates than those determined from $\mathrm{H} \alpha$ observations. Note however, that we still need to check against reddening corrections for the $\mathrm{H} \alpha$ line for all points in the sample as was pointed out by [17]. Nonetheless, significant dispersion, far from the central line will most likely remain.

\section{Discussion}

The effects of galactic interactions have so far been mostly discussed in relation to morphologi- 
cal disruption and episodes of enhanced star-formation activity. Recently we have started exploring the possibility that close galactic interactions resulting in tidal shocks that will heat gas and dust and accelerate particles. The presence of an new cosmic-ray population, in addition to already present galactic cosmic rays, can have important consequences for light element abundances [5] and the FIR-radio parameter measured in interacting, star-forming galaxies [7]. In terms of light elements, specifically lithium abundance measurements, observations in the Small Magellanic Cloud have found anomalous isotopic ratios of lithium, consistent with its ISM being exposed to additional comic-ray population, which in turn would indicate that the discrepancy between primordial lithium abundance predicted by the Big Bang Nucleosynthesis and that observed in low-metallicity halo stars, should be sought for in the form of the new physics [5]. Analysis of the FIR-radio correlation in interacting galaxies indicates that the FIR-radio parameter would vary across different interaction stages due to effects of tidal shock, causing a dispersion in the FIR-radio correlation of star-forming galaxies. Consequently this could result in the overestimate of the star-formation rate of interacting system. However, to further quantify these effect a larger sample of interacting galaxies would have to be analysed and numerical models would have to be constructed. On a final note we point out that an interesting and potentially important application of this could be found detecting interacting galaxies at high redshift by looking into their deviation from FIR-radio correlation well-established for non-interacting systems alone.

\section{Acknowledgments}

The work of TP is supported in part by the Ministry of Science of the Republic of Serbia under project numbers 171002 and 176005, and by the Provincial Secretariat for Science and Technological Development under project number 114-451-374/2015-01.

\section{References}

[1] Toomre, A., \& Toomre, J. 1972, ApJ, 178, 623

[2] Barnes, J. E., \& Hernquist, L. 1992, ARA\&A, 30, 705

[3] Sinha, M., \& Holley-Bockelmann, K. 2012, ApJ, 751, 17

[4] Sanders, D. B., \& Mirabel, I. F. 1996, ARA\&A, 34, 749

[5] Prodanović, T., Bogdanović, T., \& Urošević, D. 2013, PRD, 87, 103014

[6] Bell, A. R. 1978, MNRAS, 182, 443

[7] Donevski, D., \& Prodanovic, T. 2015, arXiv:1501.06910

[8] Reeves, H. 1970, Nature, 226, 727

[9] Howk, J. C., Lehner, N., Fields, B. D., \& Mathews, G. J. 2012, Nature, 489, 121

[10] van der Kruit, P. C. 1971, A\&A, 15, 110

[11] Yun, M. S., Reddy, N. A., \& Condon, J. J. 2001, ApJ, 554, 803

[12] Ibar, E., Cirasuolo, M., Ivison, R., et al. 2008, MNRAS, 386, 953

[13] Sargent, M. T., Schinnerer, E., Murphy, E., et al. 2010, ApJL, 714, L190

[14] Condon, J. J. 1992, ARA\&A, 30, 575 
[15] Bell, E. F. 2003, ApJ, 586, 794

[16] Corbett, E. A., Norris, R. P., Heisler, C. A., et al. 2002, ApJ, 564, 650

[17] Dopita, M. A., Pereira, M., Kewley, L. J., \& Capaccioli, M. 2002, ApJS, 143, 47

[18] Murphy, E. J. 2013, ApJ, 777, 58

[19] Helou, G., Soifer, B. T., \& Rowan-Robinson, M. 1985, ApJL, 298, L7

[20] Haan, S., Surace, J. A., Armus, L., et al. 2011, AJ, 141, 100

[21] Hopkins, A. M. 2004, ApJ, 615, 209

[22] Ivison, R. J., Greve, T. R., Dunlop, J. S., et al. 2007, MNRASs, 380, 199

[23] Seymour, N., Dwelly, T., Moss, D., et al. 2008, MNRASs, 386, 1695

[24] Carilli, C. L., Lee, N., Capak, P., et al. 2008, ApJ, 689, 883 